\title{
Estatística Descritiva utilizando o software LibreOffice Calc: experimento com um
}

\section{cubo virtual}

\author{
Descriptive Statistics using LibreOffice Calc software: experiment with a virtual cube \\ Estadísticas Descriptivas con el software LibreOffice Calc: experimente con un cubo virtual
}

Recebido: 12/01/2021 | Revisado: 14/01/2021 | Aceito: 14/01/2021 | Publicado: 17/01/2021

Edel Alexandre Silva Pontes

ORCID: https://orcid.org/0000-0002-9782-8458

Instituto Federal de Educação, Ciência e Tecnologia de Alagoas, Brasil

E-mail: edel.pontes@ifal.edu.br

Edel Guilherme Silva Pontes

ORCID: https://orcid.org/0000-0002-1138-5300

Universidade Estadual de Alagoas, Brasil

E-mail: edel@uneal.edu.br

\begin{abstract}
Resumo
Diante do advento do computador vem se discutindo em diversos congressos de educação, ciência e tecnologia novas alternativas no processo de ensino e aprendizagem eficiente de matemática. Professor e alunos interagem plenamente na busca de soluções para essa transformação educacional visando gerar modelos que valorizem o pensamento crítico, criativo e inovador. O uso das Tecnologias da Informação e Comunicação - TICs no meio escolar tem ocasionado mudanças drásticas na forma de ensinar e na forma de aprender matemática. Este artigo objetivou apresentar uma sugestão de uma aula motivacional, para alunos do ensino fundamental, utilizando medidas estatísticas descritivas no software LibreOffice Calc. Alguns questionamentos serão apresentados que venham por em dúvida a verdadeira eficiência de uma aula motivacional de Estatística na Educação Básica utilizando o Software LibreOffice Calc Esperase que propostas educacionais como essa sejam fundamentais para aproximar a escola tradicional do mundo tecnológico.
\end{abstract}

Palavras-chave: Processo de ensino e aprendizagem de matemática; Estatística descritiva; LibreOffice calc.

\begin{abstract}
With the advent of the computer, several alternatives in the process of teaching and learning mathematics have been discussed in several congresses of education, science and technology. Teacher and students interact fully in the search for solutions to this educational transformation in order to generate models that value critical, creative and innovative thinking. The use of Information and Communication Technologies - ICTs in the school environment has caused drastic changes in the way of teaching and the way of learning mathematics. This article aimed to present a suggestion of a motivational class for elementary school students, using descriptive statistical measures in the LibreOffice Calc software. Some questions will be presented that may question the true efficiency of a motivational class in Statistics in Basic Education using the LibreOffice Calc Software. Educational proposals such as this one are expected to be fundamental to bring the traditional school closer to the technological world.
\end{abstract}

Keywords: Mathematics teaching and learning process; Descriptive statistics; LibreOffice calc.

\section{Resumen}

Ante la llegada de la computadora, en varios congresos de educación, ciencia y tecnología se han debatido nuevas alternativas en el proceso de enseñanza y aprendizaje de la matemática eficiente. El profesor y los estudiantes interactúan plenamente en la búsqueda de soluciones para esta transformación educativa con el objetivo de generar modelos que valoren el pensamiento crítico, creativo e innovador. El uso de las Tecnologías de la Información y la Comunicación - TIC en el entorno escolar ha provocado cambios drásticos en la forma de enseñar y en la forma de aprender las matemáticas. Este artículo tuvo como objetivo presentar una sugerencia de una clase motivacional, para estudiantes de primaria, utilizando medidas estadísticas descriptivas en el software LibreOffice Calc. Se presentarán algunas preguntas que pueden poner en duda la verdadera eficacia de una clase motivacional de Estadística en Educación Básica utilizando el Software LibreOffice Calc Se espera que propuestas educativas como esta sean fundamentales para acercar la escuela tradicional al mundo tecnológico.

Palabras clave: Proceso de enseñanza y aprendizaje de las matemáticas; Estadísticas descriptivas; LibreOffice calc. 


\section{Introdução}

Na contemporaneidade, o uso das Tecnologias da Informação e Comunicação - TICs no ambiente escolar tem sido uma alternativa bastante presente no processo de ensino e aprendizagem de modelos matemáticos na educação básica. Para Marinheiro (2016, p.1) “Os avanços tecnológicos têm proporcionado o surgimento de diversas aplicações e equipamentos que estão cada dia mais presentes no cotidiano das pessoas, o que tem gerado mudanças procedimentais e atitudinais para lidar com tais inovações". Desta forma, o aprendiz é conduzido a desenvolver suas inúmeras habilidades, entre elas: o pensamento criativo, a intuição e o raciocínio lógico. Para Vigotski (1991) cada disciplina escolar tem uma relação particular com o curso do desenvolvimento do aluno, que altera na passagem do aluno de uma etapa para outra. A importância de criar novos meios tecnológicos no processo de ensino e aprendizagem faz acelerar o desenvolvimento cognitivo do aluno aprendiz.

$\mathrm{Na}$ sociedade atual justifica-se que os professores usem as TIC com os alunos na perspectiva de explorar novas formas de tratar e representar a informação. Isto é, deve haver um redimensionamento do papel do professor e dos alunos de modo que novas relações sejam estabelecidas a partir do potencial que as TIC proporcionam. [...] Tais aspectos possibilitam a construção de conhecimentos significativos que auxiliam no desenvolvimento de tarefas de alto nível cognitivo em todas as áreas ou níveis de modalidades de ensino (Coan, Viseu \& Moretti, 2013, p.223).

As distorções observadas na estrutura do modelo tradicional de ensino estão vinculadas ao problema do enfoque unificado e alheio ao ensino por objetivos. Em uma sala de aula, usando-se os meios tradicionais de ensino (quadro-negro e giz ou pincel) os alunos tendem a ser tratados de forma unificada e, portanto, não individualizada. Isso implica problemas na medida em que se constatam, em uma turma, alunos dotados de formas diferenciadas de aprendizagem.

Moretti (2015), Leontiev (1988), Facci (1988) afirmam que no decorrer da vida, diversas atividades têm a possibilidade de potencializar a aprendizagem e são chamadas "atividades principais", porque são as que ocorrem mais intensamente à apropriação e se constitui a principal forma de relacionamento do sujeito com a realidade. Para Vigotski (apud Morreti, 2015) que as crianças conseguem elaborar aptidões e conhecimentos socialmente disponíveis, nas quais elas conseguem interiorizar.

Na busca de encontrar resultados mais significativos no processo de ensino e aprendizagem de modelos matemáticos na educação básica, este artigo propõe uma alternativa didática bastante inovadora e motivadora: $\mathrm{O}$ uso do software LibreOffice Cal para determinação de medidas estatísticas descritivas na educação básica. “O ato de ensinar estatística na educação básica deve oportunizar condições para o aluno aprendiz gerar autonomia suficiente para estabelecer relações dos conteúdos aprendidos com os modelos de seu dia a dia” (Pontes, 2018, p.220).

As inovações tecnológicas oriundas do ultimo século, sugerem transformações nos mais variados segmentos da vida moderna. "Mudança é a palavra de ordem na sociedade atual. Os meios de produção e de serviço passam por profundas mudanças, caracterizadas como uma mudança de paradigma” (Valente et al, 1999, p.31). O educador, espectador atento deste processo, necessita de um ferramental adequado e sustentador de tais metamorfoses. "O ensino tradicional deve ser substituído por um ensino motivador aproximando o aluno de sua realidade" (Pontes, 2019a, p.203). Neste sentido, a trilogia: aluno, professor e computador vêm se configurando em dimensões consideráveis e irreversíveis. As novas tecnologias têm colocado a disposição, particularmente da comunicação e da educação, uma serie de recursos que podem contribuir significativamente para mudanças no processo de ensino e aprendizagem.

Se as escolas, de certa forma, não foram atingidas pelo advento da tecnologia digital, o mesmo não pode ser dito da vida das crianças quando estão fora da escola. Muito pelo contrário, a infância contemporânea está permeada, em alguns sentidos até definida, pela mídia moderna - através da televisão, do vídeo, dos jogos de computador, da 
Internet, da telefonia móvel, da música popular e pelo leque de commodities ligadas à mídia que formam a cultura do consumo contemporâneo (Buckkingham, 2010, p.42).

Para Pontes (2013) as novas tecnologias voltadas a área de Educação Matemática, particularmente para Tecnologia da Informação e Comunicação (TIC) possuem uma série de recursos que podem contribuir significativamente para mudanças no processo de ensino e aprendizagem de matemática. Apesar dessas TICs não estarem ainda completamente envolvidas em todo sistema educacional são elas instrumentos indispensáveis para o ensino integrado com o cotidiano do aluno aprendiz.

No cotidiano dos cidadãos, as inovações tecnológicas são incorporadas crescentemente em processos e atividades, tais como: compras online, informações e certificações públicas, consultas e movimentações bancárias, eleições, declaração do imposto de renda etc. A consequência disso é o surgimento de novas necessidades de aprendizagem nos mais diversos segmentos da sociedade e, em especial, na educação. Os professores são pressionados cada vez mais a incorporar a tecnologia para criar recursos pedagógicos informáticos, mas que não fizeram parte de sua história como alunos, nem de sua formação como professores (Jacon \& Kalhil, 2011, p.28).

Os Parâmetros Curriculares Nacionais - PCNs sugere com destaque o ensino de matemática agregada as TICs, sobretudo com a utilização dos computadores como ferramenta primordial para uma prática pedagógica mais eficiente.

As potencialidades das TIC como instrumento de trabalho, de informação e de comunicação durante a prática pedagógica são amplamente reconhecidas. Como meio de informação, as TIC permitem que os futuros professores, por um lado, pesquisem e explorem sites, links, software e documentos on-line com interesse para o ensino de Matemática e, por outro lado, selecionam recursos tecnológicos para utilizar na sala de aula. (Viseu \& Ponte, 2009, p.386).

Segundo Marin (2017) a utilização de TICs no ensino de matemática é bastante recomendado por especialistas pela circunstância que os alunos possam desenvolver diversas atividades com o uso de tabelas, gráficos e expressões algébricas de maneira mais eficiente e articulada. Para Brocardo e Mendes (2001) numa sociedade baseada na informação, na comunicação e na tecnologia, a formação básica em estatística torna-se indispensável ao cidadão pelo motivo de se qualificar a organizar, descrever, interpretar dados e tomar decisões ou fazer previsões com base nesses dados.

Os Parâmetros Curriculares Nacionais discutem a importância da introdução do estudo de estatística já nas séries iniciais, argumentando que a coleta de dados e representação de dados são fontes de situações-problema reais, envolvendo contagem, números, medidas, cálculos e estimativas que favorecem a comunicação oral e escrita (Guimarães et al,2009,p.135).

A Estatística é um ramo da matemática que tem como objetivo fazer previsões de determinado universo estudado, a partir de uma amostra significativa. O Ensino de Estatística pode ocorrer em qualquer nível de ensino, pois é um instrumento fundamental tanto nas aulas práticas como na elaboração de trabalhos escritos.

Para promover conhecimento e consciência sobre dados, é importante prover contextos relevantes para as ideias apresentadas em classe. Para os estudantes, é muito importante que eles possam perceber porque os dados foram coletados e o que o pesquisador quer fazer com eles. Os estudantes não sabem a priori por que eles precisam saber os conceitos estatísticos, o trabalho com exemplos relevantes e interessantes os fará apreciar a importância do conhecimento estatístico (Campos, Wodewotzki \& Jacobini, 2013, P.25).

Segundo Tenório, et al (2017) a utilização de softwares matemáticos educativos é extremamente eficientes para o processo de ensino e aprendizagem de estatística, pois permite que o aluno experimente construções e descubra conceitos a partir do manuseio de gráficos, tabelas e medidas. 


\section{Metodologia}

No que concerne aos procedimentos técnicos abordados para este estudo documental de natureza qualitativa foi utilizado o Software livre LibreOffice Cal. O Calc (planilhas eletrônicas) é um dos componentes do LibreOffice bastante útil para trabalhar com análise de dados numéricos e tomada de decisões. Inicialmente, foi feito um levantamento bibliográfico para avaliar os tópicos de estatística que seriam usados no software LibreOffice Calc, assegurando que os objetivos fossem alcançados de forma plena e dentro dos ambientes virtuais.

Nem sempre é fácil realizar pesquisas e um dos motivos é a falta de conhecimento sobre as metodologias e técnicas. Quando se tem o saber necessário para realizar os trabalhos pode-se alcançar a autonomia. [...] Método é o caminho para se realizar alguma coisa e quando se tem o caminho, torna-se mais fácil realizar viagens sabendo onde se está e aonde se quer chegar e como fazê-lo. [...] Os métodos qualitativos são aqueles nos quais é importante a interpretação por parte do pesquisador com suas opiniões sobre o fenômeno em estudo. (Pereira et al. 2018, p.67).

Os conteúdos de estatística relatados nesta pesquisa foram: geração de números aleatórios, média aritmética, mediana, moda, variância e desvio padrão. Observa-se que são conceitos elementares da estatística que podem ser trabalhados facilmente na Educação Básica. "É inadmissível ocorrer uma transformação científica e tecnológica no mundo atual conectado a uma grande deformidade exibida nas instituições de ensino no manejo em preparar a matemática (ou a estatística) como um instrumento indispensável para o desenvolvimento metacognitivo do sujeito envolvido" (Pontes et al. 2020, p. 21).

Para que esses conceitos sejam compreendidos de forma mais eficaz e interessante para o aluno aprendiz, este trabalho sugere uma aula motivacional sobre o estudo desses conceitos estatísticos utilizando o LibreOffice Cal através de um experimento com um cubo virtual.

O cubo é um hexaedro regular que contem seis faces, numeradas de 1 a 6 , com igual probabilidade de ocorrência. A ideia era lançar virtualmente o cubo, 10 vezes, 20 vezes e 30 vezes, de maneira a preservar uma boa fidedignidade dos dados.

De acordo com Pontes (2012), as experiências com dados apresentam resultados significativos quando o número de observações do experimento passa de três vezes a quantidade de elementos do espaço amostral. Entre vários testes em sala de aula com os poliedros de Platão, o autor observou que no caso do hexaedro regular a quantidade de lançamentos deve ser de no mínimo de dezoito repetições para uma representatividade significativa das probabilidades.

A Tabela 1 mostra toda a construção de uma aula motivadora que objetivou através da simulação de um cubo virtual, apresentar conceitos básicos estatísticos. 
Research, Society and Development, v. 10, n. 1, e34410111911, 2021

(CC BY 4.0) | ISSN 2525-3409 | DOI: http://dx.doi.org/10.33448/rsd-v10i1.11911

Tabela 1 - Etapas do experimento: Estudo Estatístico do Cubo Virtual.

\begin{tabular}{|c|c|c|}
\hline \multicolumn{3}{|c|}{ Tema da Aula: Experimento com um Dado Virtual no LibreOffice Calc } \\
\hline Etapas & Procedimento & Dispositivo \\
\hline I & $\begin{array}{l}\text { Definir os conceitos básicos das seguintes medidas } \\
\text { estatísticas: geração de números aleatórios, média } \\
\text { aritmética, mediana, moda, variância e desvio padrão. }\end{array}$ & Quadro-negro \\
\hline II & Apresentar as medidas da ETAPA I no LibreOffice Calc & Planilha do LibreOffice Calc \\
\hline & $\begin{array}{l}\text { Experiência 1: Gerar } 10 \text { números aleatórios inteiros de } 1 \text { a } \\
\text { 6. (Cubo Virtual 1) } \\
\text { Experiência 2: Gerar } 20 \text { números aleatórios inteiros de } 1 \text { a }\end{array}$ & $\begin{array}{l}\text { Utilizar a função: } \\
\text { ALETÓRIOENTRE (inferior, superior). }\end{array}$ \\
\hline III & $\begin{array}{l}\text { 6. (Cubo Virtual 2) } \\
\text { Experiência 3: Gerar } 30 \text { números aleatórios inteiros de } 1 \text { a } \\
\text { 6. (Cubo Virtual 3) }\end{array}$ & \\
\hline IV & Calcular a Média Aritmética dos três Experimentos. & $\begin{array}{l}\text { Utilizar a função: } \\
\text { MÉDIA (número 1, número 2,...). }\end{array}$ \\
\hline $\mathbf{V}$ & Calcular a Moda dos três Experimentos. & MODO (número 1, número 2,...). \\
\hline VI & Calcular a Mediana dos três Experimentos. & MED (número 1, número 2,...). \\
\hline VII & Calcular a Variância Amostral dos três Experimentos. & VARA (número 1 , número $2, \ldots$ ). \\
\hline VIII & Calcular a Desvio Padrão amostral dos três Experimentos. & DESVPADA (número 1 , número $2, \ldots$ ). \\
\hline
\end{tabular}

Fonte: Elaboração dos Autores.

Experiências em sala de aula são práticas pedagógicas fundamentais para desenvolver o intelecto do aluno envolvido. Os desafios e problemas propostos para o aprendiz o levam a desenvolver diversas técnicas para pensar e fazer matemática. A escola e o professor são os fatores primordiais para transformar seu aluno, protagonista do processo, no individuo com potencial para encarar as situações do mundo real e tecnológico. Da Silva (2017) salienta que o processo de ensino e aprendizagem envolve uma ação por parte do aluno aprendiz e que essa ação seja problematizadora e que provoque no sujeito o interesse pela busca da solução

O professor pode passar uma informação, mas verdadeiramente ensina seus alunos quando sabe converter essa informação em conhecimento, tranformando-os. Assim a verdadeira e transformadora aprendizagem é processo que começa com o confronto entre a realidade que sabemos e algo novo que descobrimos ou mesmo uma nova maneira de se encarar a realidade (Selbach et al, 2010, p,18).

A proposta da utilização do software LibreOffice Calc como ferramenta para o estudo das medidas estatísticas descritivas na educação básica deve ser analisada seguindo rigorosamente as etapas metodológicas definidas na Tabela 1. Desta maneira, cada etapa é definida:

Etapa I: Definir as medidas Estatísticas Descritivas:

Números aleatórios: Conjunto de números naturais que são gerados por um processo envolvendo um elemento de chance, isto é, são números que pertence a uma série numérica, onde cada um não pode ser previsto a partir dos outros da série. 
Média Aritmética: é o número médio das observações (amostra). É a razão entre ao soma dos valores das observações pela quantidade de observações.

Moda: é o elemento de maior frequência das observações.

Mediana: é o elemento central das observações.

Variância: é a média aritmética dos quadrados dos desvios.

Desvio Padrão: é a raiz da variância. Determina o quanto as observações estão variando em torno de sua média.

Etapa II: Apresentar as medidas estatísticas no LibreOffice Calc.

Gerar números aleatórios: utilizar a função ALEATÓRIOENTRE (inferior, superior) - Retorna um número aleatório entre os números especificados.

Determinar Média Aritmética: utilizar a função MÉDIA (número 1, número 2,...) - Retorna a média de uma amostra.

Determinar Moda: utilizar a função MODO (número 1, número 2,...) - Retorna o valor mais comum uma amostra.

Determinar Mediana: utilizar a função MED (número 1, número 2,...) - Retorna a mediana de uma amostra.

Determinar Variância: utilizar a função VARA (número 1, número 2,...) - Retorna a Variância com base em uma amostra.

Determinar desvio Padrão: utilizar a função DESVPAD (número 1, número 2,...) - Calcula o Desvio Padrão com base em uma amostra.

Etapa III: Gerar três simulações com o Cubo virtual.

Cubo virtual 1: gerar 10 números aleatórios (A2:A11). ALEATÓRIOENTRE (1,6) - Coluna A.

Cubo virtual 2: gerar 20 números aleatórios (B2:B21)., ALEATÓRIOENTRE(1,6) - Coluna B.

Cubo virtual 3: gerar 30 números aleatórios $(\mathrm{C} 2: \mathrm{C} 31)$., ALEATÓRIOENTRE(1,6) - Coluna C.

Etapa IV: Calcular a Média Aritmética.

Cubo virtual 1: MÉDIA(A2;A11) - Coluna F2.

Cubo virtual 2: MÉDIA(B2;B21) - Coluna G2.

Cubo virtual 3: MÉDIA(C2;C31) - Coluna H2.

Etapa V: Calcular a Moda.

Cubo virtual 1: MODO(A2;A11) - Coluna F3.

Cubo virtual 2: $\operatorname{MODO}(\mathrm{B} 2 ; \mathrm{B} 21)$ - Coluna G3.

Cubo virtual 3: MÉDIA(C2;C31) - Coluna H3.

Etapa VI: Calcular a Mediana.

Cubo virtual 1: MED (A2;A11) - Coluna F4.

Cubo virtual 2: MED (B2;B21) - Coluna G4.

Cubo virtual 3: $\operatorname{MED}(\mathrm{C} 2 ; \mathrm{C} 31)-$ Coluna H4.

Etapa VII: Calcular a Variância.

Cubo virtual 1: VARA (A2;A11) - Coluna F5.

Cubo virtual 2: VARA (B2;B21) - Coluna G5.

Cubo virtual 3: VARA(C2;C31) - Coluna H5.

Etapa VIII: Calcular o Desvio Padrão.

Cubo virtual 1: DESVPAD (A2;A11) - Coluna F6.

Cubo virtual 2: DESVPAD (B2;B21) - Coluna G6.

Cubo virtual 3: DESVPAD (C2;C31) - Coluna H6. 
Research, Society and Development, v. 10, n. 1, e34410111911, 2021

(CC BY 4.0) | ISSN 2525-3409 | DOI: http://dx.doi.org/10.33448/rsd-v10i1.11911

O Quadro 1 apresenta os resultados do experimento sugerido para a aula motivacional. Campos et al. (2013) afirma que é bastante salutar focar muito mais aulas nos processos estatísticos, em interpretações de dados e em reflexões dos resultados alcançados, do que na valorização de fórmulas e de cálculos.

Quadro 1: Simulação de um Cubo (Dado) Virtual e as medidas estatísticas descritivas.

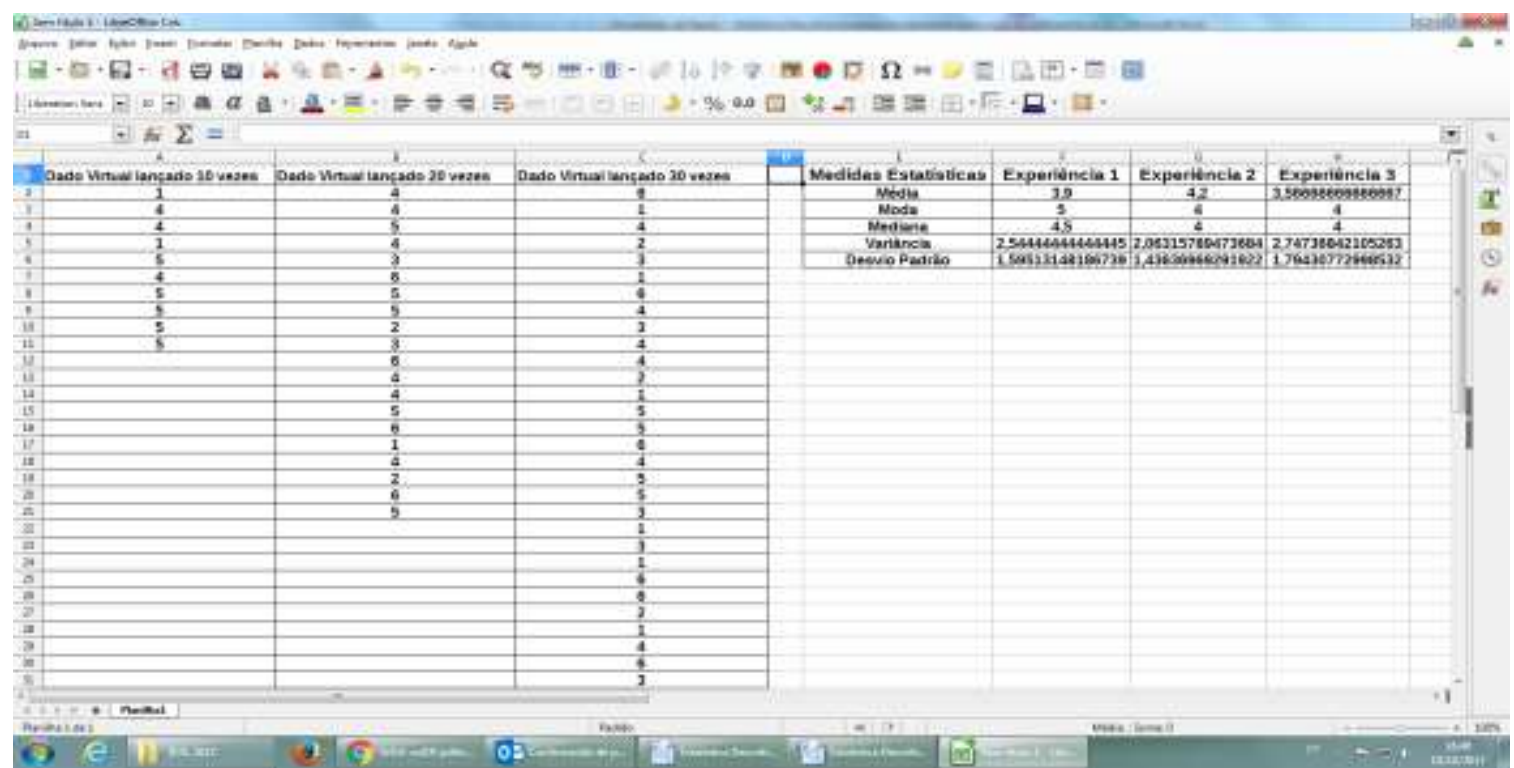

Fonte: Elaboração dos Autores.

Faz-se necessário, para um ensino de estatística na educação básica, desenvolver alguns aspectos, tais como, "incentivar os alunos a analisar e interpretar resultados, valorizar a escrita, promover julgamentos sobre a validade das ideias e das conclusões, fomentar a criticidade e cobrar dos alunos o seu posicionamento perante os questionamentos" (Campos et al, 2013, p.63).

Observa-se que se o aluno aprendiz seguir rigorosamente todas as etapas do experimento indicado, para a aula motivacional, o resultado alcançado será extremamente satisfatório, pois é uma atividade que requer do sujeito envolvido apenas saber manusear um computador e vontade de aprender.

\section{Resultados e Discussão}

Percebe-se que alguns questionamentos podem surgir que venham por em dúvida a verdadeira eficiência da aula motivacional de Estatística na Educação Básica utilizando o Software LibreOffice Calc, entre elas:

a. A escola tem equipamentos necessários para esse tipo de atividades?

b. Os professores de matemática estão capacitados para essa prática motivacional?

c. É necessário repensar o currículo de matemática da educação básica para se adequar ao novo modelo de ensino?

Apesar das inúmeras iniciativas governamentais, a introdução da Informática Educacional nas escolas públicas aconteceu de forma lenta e com certa indefinição do seu real objetivo. Em algumas escolas, os dirigentes e os professores interessavam-se em incrementar os currículos com o ensino básico dos conceitos da informática; outros buscavam utilizá-los como recurso no processo de ensino-aprendizagem e, nesse conflito, até hoje, os professores não assumiram, de fato e de maneira adequada, o uso da Informática em seu fazer pedagógico (Gomes \& Moita, 2016, p. 156). 
Em relação à primeira indagação (A escola tem equipamentos necessários para esse tipo de atividades?), nota-se que o mundo evoluiu e a escola atual deve está pronta para se adequar as novas tecnologias. A escola e o computador são indissociáveis no contexto atual da educação básica e, é inadmissível se falar de um sem enunciar o outro. Além disso, o uso de Softwares educativos estimula o aluno a compreender o modelo apresentado e faz despertar sua criatividade e raciocínio. A tarefa de melhorar o sistema educacional brasileiro exige um profundo esforço governamental para equipar as escolas públicas de educação básica com laboratórios de informática instalados e em pleno funcionamento.

O professor como agente mediador dos conhecimentos, precisa saber utilizar o software e explorar ao máximo as possibilidades de uso. Ao redor do mundo inteiro, as crianças entraram em um mundo mágico de aprendizagem e de alegria com os computadores. Elas utilizam os computadores para escrever, para desenhar, para se comunicar e para obter informações (Gomes \& Moita, 2016, p. 165).

Em relação à segunda indagação (Os professores de matemática estão capacitados para essa prática motivacional?), tendo resolvido a primeira, se faz necessário capacitar professores para utilizarem as ferramentas computacionais disponíveis, nos laboratórios de informática, de forma prática e eficiente. O professor desempenha uma função insubstituível no processo educativo e para isso precisa está estimulado e totalmente preparado para desempenhar esse papel de mediador do conhecimento. Gomes e Moita (1999) retrata que a escola deveria ter um projeto permanente e contínuo na formação de professores para atuarem na área de Educação e Tecnologia, o que agregaria melhores condições de trabalho.

Quando o computador transmite informação para o aluno, o computador assume o papel de máquina de ensinar, e a abordagem pedagógica é a instrução auxiliada por ele. Essa abordagem tem suas raízes nos métodos tradicionais de ensino, porém, em vez da folha de instrução ou do livro de instrução, é usado o computador. Os softwares que implementam essa abordagem são os tutoriais e os de exercício-e-prática (Valente et al, 1999, p.12)

Em relação à terceira indagação (É necessário repensar o currículo de matemática da educação básica para se adequar ao novo modelo de ensino?), o currículo de matemática na educação básica deve constantemente ser realinhado com as necessidades e transformações do mundo moderno. A informática educativa atrelada as diversas áreas de formação básica fortalecem as condições do aluno de construir seu conhecimento.

\section{Considerações Finais}

Este trabalho apontou uma alternativa para definir medidas estatísticas, no ensino fundamental, utilizando o software LibreOffice Calc. Essas são práticas bastante motivadoras e geram no aluno aprendiz estímulos necessários para desenvolver maior criatividade no processo de aprender modelos matemáticos. Para Pontes (2013) nos últimos anos vem se discutindo a relação entre o ensino de matemática com o desenvolvimento da inteligência, isto é, quanto melhor as práticas educacionais e mais variadas às oportunidades tecnológicas na escola, maior o desenvolvimento da inteligência.

A utilização das Tecnologias da Informação e Comunicação no cotidiano escolar permite aos alunos desenvolver novos conhecimentos por meios de atividades interativas com o computador. A condição suficiente e necessária para que o professor possa programar o ensino de estatística na educação básica é ter grande capacidade e sensibilidade de transformar sua pratica pedagógica de maneira que possa eficientemente acordar seu aluno aprendiz para a interpretação de dados e tomada de decisão. Lopes (2008) indaga que todo processo de ensino e aprendizagem que envolva o pensamento estatístico possibilita formar um aluno com maior poder de análise e criticidade diante de dados e índices.

A formação do educador matemático que atua ou atuará na educação básica deve prever um processo de ensino e aprendizagem de conteúdos que ocorra através da resolução de problemas, simulações e experimentos, os quais 
Research, Society and Development, v. 10, n. 1, e34410111911, 2021

(CC BY 4.0) | ISSN 2525-3409 | DOI: http://dx.doi.org/10.33448/rsd-v10i1.11911

permitam ao profissional construir conhecimentos, à medida que estabelecer relações com informações adquiridas e com o domínio de diferentes linguagens e formas de expressão (Lopes, 2008, p.71).

Está sugestão como prática educacional no processo de ensino e aprendizagem de matemática possibilita ao professor facilitador e ao aluno aprendiz desenvolver novas habilidades no intuito de fortalecer o pensamento crítico e o raciocínio lógico matemático.

Um aluno, por exemplo, pode memorizar uma tabuada ou manusear uma calculadora, mas aprende matemática quando, ajudado por um professor ou um colega, descobre uma nova maneira de perceber coisas que antes não percebia. Nesse caso, o aluno não conquistou uma nova informação, mas aprendeu outra maneira de olhar o que sempre olhava e, assim, essa nova maneira o transformou (Selbach et al, 2010, p,16).

A proposta visa harmonizar a escola meramente tradicional e limitada a repassar e reproduzir mecanicamente conhecimento com o mundo tecnológico. Para Valente et al (1999) a transformação pedagógica que todos esperam é a passagem de uma educação de transmissão de conhecimento, para a criação de um modelo de ambientes de aprendizagem de forma que o próprio aluno construa seu conhecimento.

O professor deve ter consciência de seu papel como mediador do processo e que esteja sempre pronto a retratar novas técnicas e métodos que possam minimizar as defasagens entre a teoria abstrata de matemática com a prática do dia a dia do aprendiz. Em contra partida, o aluno aprendiz, o soldado do conhecimento, deve acreditar em seu potencial e ficar sempre disponível a desenvolver formas de adquirir novos conhecimentos (Pontes, 2019b, 2019, p.10).

Espera-se que outras experiências possam ser sugeridas como modelos transformadores de uma educação pautada no ser engenhoso, produtivo e que esteja pronto para enfrentar a era tecnológica.

\section{Referências}

Brocardo, J., \& Mendes, F. (2001). Processos usados na resolução de tarefas estatísticas. Quadrante, 10, 33-58.

Buckingham, D. (2010). Cultura digital, educação midiática e o lugar da escolarização. Educação \& Realidade, 35(3), 37-58.

Campos, C. R., Wodewotzki, M. L. L., \& Jacobini, O. R. (2013). Educação Estatística: teoria e prática em ambientes de modelagem matemática. Autêntica.

da Silva, A. J. N., do Nascimento, A. M. P., \& Muniz, C. A. (2017). O necessário olhar do professor sobre a produção matemática das crianças nos Anos Iniciais. Educação Matemática em Revista, 48-55.

Facci, M. G. D. (2004). A periodização do desenvolvimento psicológico individual na perspectiva de Leontiev, Elkonin e Vigostski. Cadernos Cedes, 24(62), 64-81.

Gomes, L. L., \& Moita, F. O. (2016). uso do laboratório de informática educacional: partilhando vivências do cotidiano escolar. SOUSA, RP et al., orgs. Teorias e práticas em tecnologias educacionais [online]. Campina Grande: EDUEPB, 151-174.

Borba, R., \& Guimarães, G. (2009). A pesquisa em educação matemática: repercussões na sala de aula: Cortez, 2009.

Jacon, L. D. S. C., \& Kalhil, J. B. (2011). O professor formador e as competências em tecnologia de informação e comunicação: um estudo sobre quais recursos computacionais estes profissionais utilizam na elaboração do seu material didático. Amazônia: Revista de Educação em Ciências e Matemáticas, 8(15), 27-44.

Leontiev, A. N. (1988). Os princípios psicológicos da brincadeira pré-escolar. Linguagem, desenvolvimento e aprendizagem, 5, $119-142$.

Marin, D. (2012). Professores universitários que usam a tecnologia de informação e comunicação no ensino de matemática: quem são eles? University teachers to use technology information and communication in the teaching of mathematics: who are they?. Revista Eletrônica de Educação Matemática, 7(1), 62-77.

Coan, L. G. W., Viseu, F., \& Moretti, M. T. (2013). As TIC no ensino de Matemática: a formação dos professores em debate. REVEMAT., $222-244$.

Lopes, C. E. (2008). O ensino da estatística e da probabilidade na educação básica e a formação dos professores. Cadernos Cedes, 28(74), 57-73.

Marinheiro, F., Silva, I., Madeira, C., Cordeiro, S., Souza, D., Costa, P., \& Fernandes, G. (2016). Ensinando crianças do ensino fundamental a programar computadores com o auxílio de jogos digitais. 
Research, Society and Development, v. 10, n. 1, e34410111911, 2021 (CC BY 4.0) | ISSN 2525-3409 | DOI: http://dx.doi.org/10.33448/rsd-v10i1.11911

Moretti, V. D., \& de Souza, N. M. M. (2015). Educação Matemática nos anos iniciais do Ensino Fundamental: princípios e práticas pedagógicas. Cortez Editora

Pereira, A. S., Shitsuka, D. M., Parreira, F. J., \& Shitsuka, R. (2018). Metodologia da pesquisa científica.

Pontes, E. A. S. (2013). Hipermat-Hipertexto Matemático: Uma ferramenta no ensino-aprendizagem da matemática na educação básica. Revista Psicologia \& Saberes, 2(2).

Pontes, E. A. S. (2017). Os números naturais no processo de ensino e aprendizagem da matemática através do lúdico. Diversitas Journal, 2(1), 160-170.

Pontes, E. A. S. (2018). Algumas Considerações Teóricas No Ensino De Estatística Na Educação Básica De Maneira Contextualizada. Revista Eixo, 7(3), 227237.

Pontes, E. A. S. (2019). A Capacidade de Gerar Soluções Eficientes e Adequadas no Processo Ensino e Aprendizagem de Matemática. Revista Psicologia \& Saberes, 8(10), 193-205.

Pontes, E. A. S. (2019). Conceptual questions of a teacher about the teaching and learning process of mathematics in basic education. Research, Society and Development, 8(4), 784932.

Pontes, E. A. S., de Miranda, J. R., dos Santos, J. F., Ferreira, M. B., dos Santos Soares, M., dos Santos, R. C., \& Sobrinho, R. D. K. F. (2020). Investigação Experimental de um Produto Educacional: um jogo matemático desenvolvido a partir do conceito intuitivo de probabilidades. RACE-Revista de Administração do Cesmac, 7, 20-30.

Pontes, E. G. (2012). Tecnologias para o aprendizado da estatística e probabilidades em cursos de nível superior. Maceió: QGráfica.

Schechter, R. (2006). BrOffice. org 2.0-Calc e Writer. Elsevier Brasil.

Selbach, Simone, et al. (2010). Matemática e didática: Vozes.

Severino, A. J. (2017). Metodologia do trabalho científico. Cortez editora.

Tenório, A., do Nascimento, C. P., \& Tenório, T. (2017). Uso do software Geogebra, blog, e-mail e whatsapp no estudo de Matemática. Revista Tecnologias na Educação, ano, 9.

Vygotsky, L. S. (1988). Aprendizagem e desenvolvimento intelectual na idade escolar. Linguagem, desenvolvimento e aprendizagem, $10,103-117$.

Viseu, F., \& Ponte, J. P. D. (2009). Desenvolvimento do conhecimento didáctico do futuro profes sor de Matemática com apoio das TIC's. Revista latinoamericana de investigación en matemática educativa, 12(3), 383-413. 